\title{
Proposed ordering of textured spin singlets in a bulk infinite-layer nickelate
}

\author{
Hyo-Sun Jin, ${ }^{1}$ Warren E. Pickett $\odot,{ }^{2, *}$ and Kwan-Woo Lee $\oplus^{1,3, \dagger}$ \\ ${ }^{1}$ Division of Display and Semiconductor Physics, Korea University, Sejong 30019, Korea \\ ${ }^{2}$ Department of Physics, University of California, Davis, California 95616, USA \\ ${ }^{3}$ Department of Applied Physics, Graduate School, Korea University, Sejong 30019, Korea
}

(Received 23 June 2020; revised 6 July 2020; accepted 13 July 2020; published 5 August 2020)

\begin{abstract}
The infinite-layer structure nickelate $\mathrm{Ba}_{2} \mathrm{NiO}_{2}(\mathrm{AgSe})_{2}$ (BNOAS) with $d^{8} \mathrm{Ni}$ ions and a peculiar susceptibility $\chi(T)$, synthesized at high pressure, is studied with correlated density functional methods. The overriding feature of the calculations is a violation of Hund's rule coupled with complete but unconventional spin-orbital polarization, leading to an unexpected low-spin ${ }^{1} B_{1}$, "off-diagonal singlet" textured by an internal orbital structure of compensating $d_{x^{2}-y^{2}}^{\uparrow}$ and $d_{z^{2}}^{\downarrow}$ spins. This unconventional configuration has a lower energy than conventional high-spin or low-spin alternatives. An electronic transition is obtained at a critical Ni-O separation $d_{c}^{\mathrm{Ni}-\mathrm{O}}=2.03 \AA$, which corresponds closely to the observed critical value of $2.00-2.05 \AA$, above which $\mathrm{Ni}$ becomes magnetic in square planar $\mathrm{NiO}_{2}$ compounds. We propose scenarios for the signature of magnetic reconstruction in $\chi(T)$ at $T_{m}=130 \mathrm{~K}$ without any Curie-Weiss background (no moment) that invokes ordering of $\mathrm{Ni} d^{8}$ moieties that are largely this generalized Kondo singlet. Because hole states are primarily Se $4 p$ rather than $\mathrm{O} 2 p$, the usual issue of Mott insulator versus charge transfer insulator is supplanted by a character in which electrons and holes are separated in real space. The underlying physics of this system is modeled by a Kondo sieve spin model (two-dimensional Kondo necklace) of a "Kondo" $d_{z^{2}}$ spin on each site, coupled to a $d_{x^{2}-y^{2}}$ spin that is itself strongly coupled to neighboring like-spins within the layer. The observed magnetic order places BNOAS below the quantum critical point of the Kondo sieve model, providing a realization of the long-range ordered near-singlet weak antiferromagnetic phase. We propose electron doping experiments that would drive the system toward a $d^{9-\delta}$ configuration and possible superconductivity with a similarity to the recently reported hole-doped infinite-layer cuprate $\mathrm{Ba}_{2} \mathrm{CuO}_{3.2}$ that superconducts at $73 \mathrm{~K}$.
\end{abstract}

DOI: 10.1103/PhysRevResearch.2.033197

\section{BACKGROUND}

Layered nickelate materials have attracted attention for some decades primarily due to the similarities they provide to the layered cuprates that display high-temperature superconductivity $[1,2]$. A number of layered nickelates with noninteger formal valences have been synthesized, showing a great variety of magnetic and spin/charge ordering behavior that is now understood [3-5], but no superconductivity. However, the recent discovery [6] and verification [7] of superconductivity in thin films of strained, hole-doped $\mathrm{NdNiO}_{2}$ has reinvigorated interest in layered nickelates. The focus has been on $d^{9}$, spin-half layers, due to their similarity to the cuprates, which may be (hole or electron) doped to produce high-temperature superconducting (HTS) phases. The complementary route to superconductivity, not formerly taken seriously, may arise in compounds with a $\mathrm{Ni} d^{8}$ configuration that approach the same $d^{9}$ regime upon electron doping. The $d^{8}$ configuration

\footnotetext{
*wepickett@ucdavis.edu

†mckwan@korea.ac.kr
}

Published by the American Physical Society under the terms of the Creative Commons Attribution 4.0 International license. Further distribution of this work must maintain attribution to the author(s) and the published article's title, journal citation, and DOI. brings with it the competition between low-spin and high-spin configurations, which is expected to depend on strain applied to the $\mathrm{NiO}_{2}$ layer by other components of the compound as well as crystal-field splittings, and may or may not promote HTS.

The report of synthesis of a new layered nickelate system indicates further anomalous properties of layered nickelates. Matsumoto and collaborators have reported the synthesis [8], under $7 \mathrm{GPa}$ pressure at $850{ }^{\circ} \mathrm{C}$, of $\mathrm{Ba}_{2} \mathrm{NiO}_{2} \mathrm{Ag}_{2} \mathrm{Se}_{2}$ (BNOAS) containing an "infinite-layer" $\mathrm{NiO}_{2}$ sublattice without apical oxygen sites but with an anomalously large $\mathrm{Ni}-\mathrm{O}$ separation of $2.10 \AA$. The susceptibility $\chi(T)$ is peculiar and unexplained. In spite of no Curie-Weiss term in the otherwise constant $\chi(T)$, a peak occurs at $T_{m}=130 \mathrm{~K}$ with $30 \mathrm{~K}$ full width before returning to its original value. Field cooling below $T_{m}$ increases the susceptibility below $T_{m}$, and weak magnetic peaks in powder neutron diffraction [8] at $5 \mathrm{~K}$ have been interpreted as indicating $\left(\frac{1}{2}, \frac{1}{2}, 0\right)$ antiferromagnetic (AFM) order, with the best fit to a weak structure in the diffraction data suggesting $S=1 \mathrm{Ni}$ spins. This interpretation, however, seems inconsistent with the peculiar "spinless" behavior of $\chi(T)$. BNOAS was discussed as insulating but no conductivity data on the powder samples were presented.

A nonmagnetic ground state is observed in other square planar $d^{8}$ nickelates [8,9], viz., $\mathrm{BaNiO}_{2}$ [10] and monovalent $\mathrm{LaNiO}_{2}$ (see references in Ref. [11]), so BNOAS is an anomalous case. The isovalent sister strontium compound 
SNOAS with a smaller lattice constant is more conventional, showing Curie-Weiss behavior above $150 \mathrm{~K}$ characteristic of an $S=1$ moment and strong AFM magnetic coupling (Weiss $\theta=-158 \mathrm{~K}) \cdot \chi(T)$ indicates that SNOAS undergoes some type of magnetic order around $50 \mathrm{~K}$. Matsumoto et al. ascribe the differences between the two compounds to the different $\mathrm{Ni}-\mathrm{O}$ bond lengths (that of BNOAS being unusually large), which could tip the balance between high-spin and low-spin states.

Formal valence counting, confirmed by calculations presented below, proceeds as $\left(\mathrm{Ba}^{2+}\right)_{2} \mathrm{Ni}^{2+}\left(\mathrm{O}^{2-}\right)_{2}\left(\mathrm{Ag}^{1+}\right)_{2}\left(\mathrm{Se}^{2-}\right)_{2}$, giving $\mathrm{Ni}$ a $d^{8}$, two-hole configuration. A major issue is the relative importance of low-spin $S=0$ and high-spin $S=1$ states of the ion, and (we will propose) the nature of the $S=0$ ion. As mentioned, most known layered $d^{8}$ nickelates are nonmagnetic [8,9], $S=0$, so the magnetic behavior (even without its strangeness) of these new nickelates presents new physics. Building on the underlying magnetism, one can anticipate that electron doping, moving the $\mathrm{Ni}$ formal valence toward the $d^{9}$ configuration, might induce superconductivity as has been found in the $(\mathrm{Nd}, \mathrm{Sr}) \mathrm{NiO}_{2}$ system.

Our main result here is the discovery of a distinct ionic configuration, an "off-diagonal singlet" (ODS) with internal magnetic and orbital textures in spite of a vanishing magnetic moment. A secondary finding is a phase boundary at a critical $\mathrm{Ni}-\mathrm{O}$ separation of $2.03 \AA$ that concurs with the separation of low-spin and high-spin $\mathrm{Ni}$ ions in square planar $\mathrm{NiO}_{2}$ layered compounds. After presenting the procedures in Sec. II, we analyze the resulting electronic and magnetic structures of BNOAS in Sec. III, and briefly provide some results for applied strain and analysis of the ODS in Sec. IV. In Sec. V we introduce a minimal spin model for this compound which we feel provides a platform for accounting for the peculiar behavior of $\chi(T)$. Section VI provides a discussion of the various findings, with a brief summary provided in Sec. VII.

\section{STRUCTURE AND PROCEDURES}

These compounds, which can be synthesized with several $3 d$ ions, have the body-centered-tetragonal (bct) structure (I4/mmm, No. 139), pictured in Fig. 1(a). In the bct structure, the $\mathrm{Ni}, \mathrm{O}$, and $\mathrm{Ag}$ ions sit at high-symmetry $2 a(0,0,0), 4 c$ $(0,1 / 2,0)$, and $4 d(0,1 / 2,1 / 4)$ sites, respectively. The $\mathrm{Ba}$ and $\mathrm{Se}$ ions lie on $4 e(0,0, z)$ sites with $z=0.4136$ and 0.1606 , respectively. The $\mathrm{NiO}_{2}$ layers with a large separation of $10 \AA$ form a two-dimensional square lattice without apical oxygen, as in the $d^{9}$ nickelates $\mathrm{NdNiO}_{2}$ and $\mathrm{LaNiO}_{2}$ [12-14]. The $(\mathrm{AgSe})_{2}$ layer provides some $\hat{z}$-axis coupling.

An intriguing feature of the structure is that the $(\mathrm{AgSe})_{2}$ substructure is the same as that of the FeSe-type superconductors. The overall structure is reminiscent of many cuprate and Fe-based superconductors: electronically active $\left(\mathrm{NiO}_{2}\right)$ layers separated by inactive "spacer" layers. The strain supplied by the $(\mathrm{AgSe})_{2}$ layers results in an anomalous elongation of the Ni-O bond length to 2.10 (2.05) $\AA$ for BNOAS (SNOAS), about $10 \%$ (6\%) larger than in $\mathrm{CaCuO}_{2}$ and also other layered $d^{8}$ nickelates. The $(\mathrm{AgSe})_{2}$ layers, however, are insulating and relatively inert in these compounds. (a)

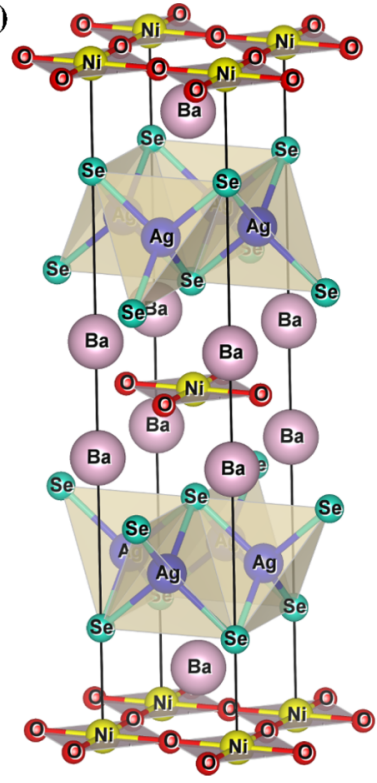

(b)

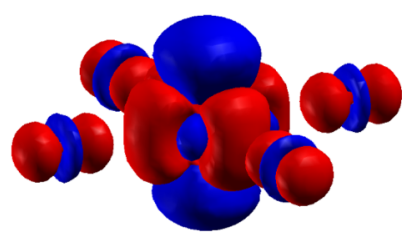

FIG. 1. (a) bct structure of $\mathrm{Ba}_{2} \mathrm{NiO}_{2}(\mathrm{AgSe})_{2}$. The layered square lattice of $\mathrm{Ni}$ ions without apical oxygen ions forms a single "infinitelayer" structure of the $\mathrm{NiO}_{2}$ layer analogous to the bulk layers of $\mathrm{CaCuO}_{2}$ and $\mathrm{NdNiO}_{2}$. Subsequent layers have a large interlayer "spacer layer" distance of about $10 \AA$, leading to strong two dimensionality in many respects. (b) Spin density plot of the spin singlet state at an isovalue of $0.015 e / \AA$ ( $U=7 \mathrm{eV}$ with WIEN2K). Red (blue) denotes spin-up (spin-down) character. This combination comprises the orbitally off-diagonal singlet state $d_{z^{2}}^{1 \downarrow}-d_{x^{2}-y^{2}}^{1 \uparrow}$. Hybridization with the planar oxygens is apparent.

Our calculations were performed with the experimental crystal structure, using the BNOAS lattice constants $a=$ $4.2095 \AA$ and $c=19.8883 \AA$, obtained from the neutron diffraction measurement at $5 \mathrm{~K}$ [8], except for a few cases where smaller planar lattice constants $a$ were used to probe the dependence of the Ni-O separation and identify a critical separation. We have compared results from two all-electron, full potential density functional theory (DFT) codes, FPLO14 [15] and WIEN2K [16], the former being an atomic orbital basis code. The latter is a linearized augmented plane-wave code that is considered to give the most precise solutions of the quantities that arise in DFT. In many tests these two codes have produced results that are equivalent within the accuracy of the underlying DFT exchange-correlation functionals.

FPLO is a local orbital-based DFT code with built-in atomic orbitals, particularly adapted to characterizing the electronic and magnetic structures of transition metal compounds [17]. In WIEN $2 \mathrm{~K}$, the basis size was determined by $R_{\mathrm{mt}} K_{\max }=7$ with augmented-plane-wave sphere radii $R_{\mathrm{mt}}$ (in a.u.): $\mathrm{Ni}$, 2.13; O, 1.83; and 2.50 for $\mathrm{Ag}$, Se, and Ba. For both codes, the Brillouin zone was sampled by a dense $k$ mesh of $20 \times$ $20 \times 20$ to check the energetics carefully.

We utilize the generalized gradient approximation (GGA) [18] plus Hubbard $U$ method $(\mathrm{GGA}+U)$ [19], with the double-counting term of the so-called around mean-field scheme $[19,20]$ as implemented in rotationally invariant fashion in the two codes we use. We note that, since $U$ and $J$ are applied to the "atomic orbital" (which is not uniquely 
defined) in different ways by the two codes, results differ somewhat. An important point of our work is that these two codes give qualitatively similar results for which the quantitative differences are unimportant.

To probe correlation effects in the GGA $+U$ calculations, we have at times varied $U$ in the range $1-9 \mathrm{eV}$, always with the fixed Hund's spin coupling $J=0.7 \mathrm{eV}$. Varying $U$ is accepted practice. There is no specific value to use for $\mathrm{Ni}$ (or any specific atom), because the large screening of the atomic value depends on the charge state, on the local environment, and on the conducting (or not) behavior of the system, the latter two providing screening. Small values are more appropriate for conducting $\mathrm{LaNiO}_{3}$, larger values for highly insulating NiO. Values of $U \approx 4-8 \mathrm{eV}$ have been applied in different contexts [2,21-23] (and see references in Ref. [11] for $\mathrm{LaNiO}_{2}$ ). For BNOAS, which undergoes a metal-insulator transition in our calculations as $U$ increases, values in the 5-7 eV range seem most physical. It is common practice to check the sensitivity of $\mathrm{DFT}+U$ results for sensitivity to the value of $U$. Our results are not sensitive to $U$ within the 5-7 eV range, or even higher.

\section{ELECTRONIC AND MAGNETIC STRUCTURE}

We begin by announcing that our results are unexpected and rather startling. The formal $d^{8}$ configuration conventionally suggests that the possibilities are (i) a high-spin $S=1 \mathrm{Ni}$ ion, with holes in both the $d_{x^{2}-y^{2}}$ and $d_{z^{2}}$ minority orbitals, or (ii) a nonmagnetic low-spin $S=0$ state with $d_{x^{2}-y^{2}}$ of both spins unoccupied. We find another, (iii) an off-diagonal singlet (ODS), with holes of opposite spins in the $d_{x^{2}-y^{2}}$ and $d_{z^{2}}$ orbitals ( ${ }^{1} B_{1}$ symmetry), with schematic energy level diagrams at the GGA level presented in Fig. 2(c). (The $t_{2 g}$ states are nearly degenerate at the GGA level.) The ODS is described and analyzed in Sec. III D.

There are unusual features of the spin density, pictured in Fig. 1(b). Distinctive features are the following: (1) While the net moment is exactly zero (singlet $S=0$ ), there remains full spin polarization of the two orbitals, with a substantial magnetic energy benefit. This specifies a singlet [24] with an internal texture, which is orbital polarization. (2) There is full spin-orbital polarization, unlike what occurs in some insulating nickelates. (3) This singlet is a pure spin analog of the nonmagnetic $\mathrm{Eu}^{3+} f^{6}$ ion with $S=3, L=3, \mathcal{J}=$ $|L-S|=0$, a singlet which nevertheless has large spin and orbital polarization [25-27]. (4) It violates Hund's first rule, without apparent cause (such as crystal-field splitting). (5) It gains Coulomb energy by remaining maximally anisotropic (fully orbitally polarized) within the two-orbital subspace. Properties related to features (1), (2), and (5) were discovered in $\mathrm{DFT}+U$ studies [28] of $\mathrm{MnO}$ under pressure. We will return to this comparison in Sec. VI.

\section{A. Energetics and moments}

The gap values of ODS, FM, and AFM states versus $U$ are shown in Fig. 2(a). Not surprisingly, the gap opens for much smaller $U=1 \mathrm{eV}$ for AFM order due to its smaller bandwidth; for the ODS the gap opens only at $U=5 \mathrm{eV}$. In all cases the gap remains small for a transition metal oxide,
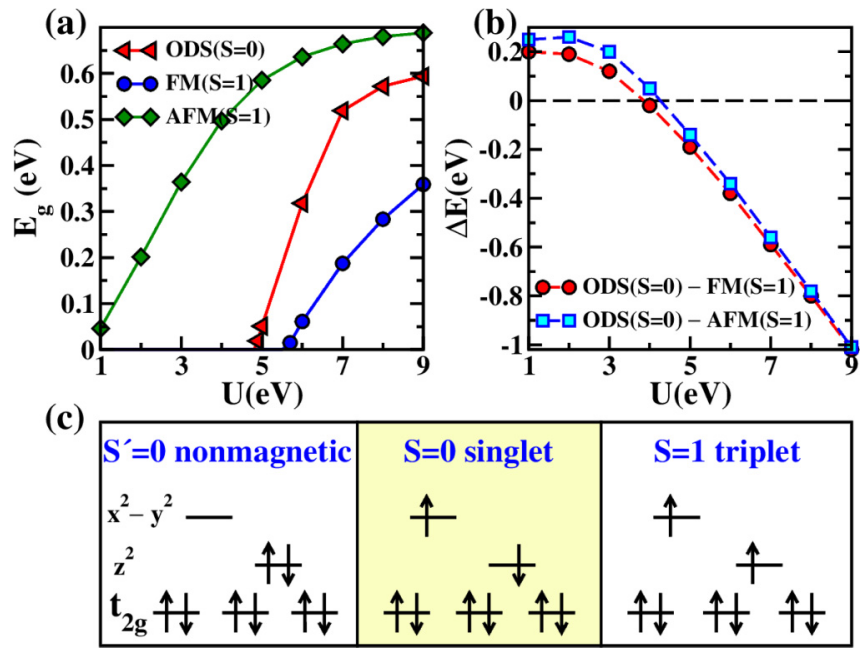

FIG. 2. Top: Variations of (a) energy gaps and (b) relative energies among ODS $(S=0)$, FM $(S=1)$, and $\operatorname{AFM}(S=1)$ ionic states and alignments, as the Coulomb repulsion $U$ is varied in the $1-9 \mathrm{eV}$ range, from FPLO. Above $U_{c}=4 \mathrm{eV}$, where the spin-up $d_{z^{2}}$ orbital is nearly emptied, the ODS is energetically favored over the other states. The physical range of $U$ is usually considered to be in the 5-7 eV range. Bottom: Schematic energy level diagrams for the states of the Ni ion; arrows indicate the spins of occupied orbitals, with the ODS in the center panel.

even for $U=9 \mathrm{eV}$. Next, we compare energies of the FM and AFM states relative to the ODS state in Fig. 2(b). The nonmagnetic (NM) state (trivial $S=0{ }^{1} A$ singlet) lacks spin polarization energy and can be neglected. Both FM and AFM ordered states have $S=1 \mathrm{Ni}$ ions with their Hund's rule energy gains, with AFM being slightly favored over FM with a difference that decreases with increasing $U$. The ODS state, which is spin-orbital polarized but $S=0$, above $U_{c}=4 \mathrm{eV}$ becomes favored over both states having $S=1$ ions, with the energy difference growing rapidly as $U$ increases. The Hund's exchange energy is overcome by other energy changes, as discussed in Sec. VID and the Appendix.

The values of the FM and AFM moments at GGA level are $\sim 1.1 \mu_{B}$, as shown in the large lattice constant $a$ region of the upper panel of Fig. 3. Including $U$, these grow (not shown) to around $1.9 \mu_{B}$, a value that is characteristic of $S=1$ reduced somewhat by hybridization. To emphasize: For the ODS ions, the orbital moment is around $0.16 \mu_{B}$ while the net spin moment vanishes.

\section{B. Effect of strain}

Matsumoto and coauthors [8] have drawn a connection between the Ni-O bond length and the low-spin and high-spin states in layered $d^{8}$ compounds, identifying a critical separation in the 2.00-2.05 $\AA$ range and ascribing the transition to crystal-field splitting. This critical distance led us to study the lattice constant dependence, taking the FM state as an example. We performed the corresponding calculations, with results shown in Fig. 3 using FPLO within GGA (WIEN2K results are similar). As $a$ is decreased from 4.2 to $3.9 \AA$ with the cell volume fixed and internal parameters of $\mathrm{Ba}$ and Se being optimized (the observed value is $a=4.21 \AA$ ), an 


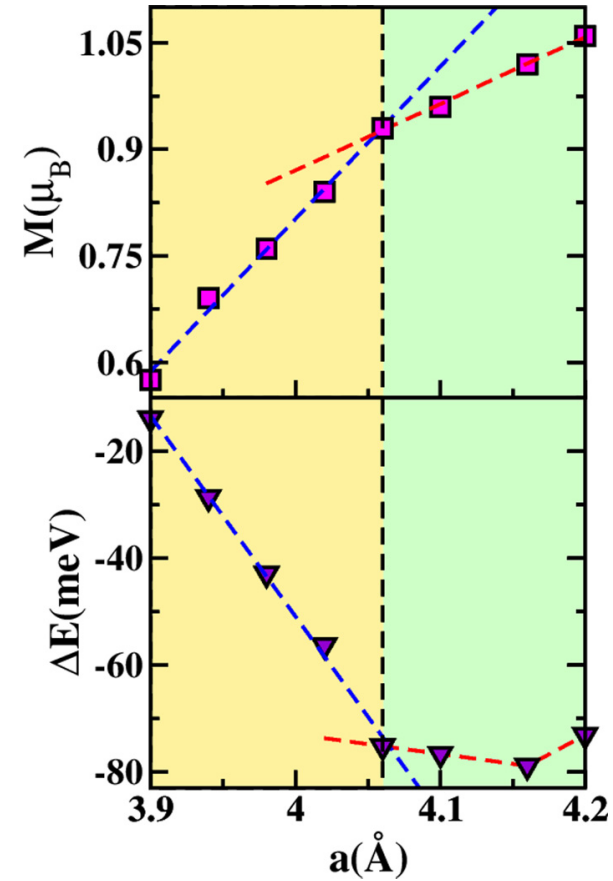

FIG. 3. Variations of (top) the Ni moment and (bottom) the energy difference $\Delta E=E_{\mathrm{FM}}-E_{\mathrm{NM}}$ between FM and NM states, as the $a$ lattice constant is varied at constant volume. From FPLO within GGA. Both the moment and energy difference display critical changes as the gap closes below a Ni-O separation of $d_{c}^{\mathrm{Ni}-\mathrm{O}}=a / 2=$ $2.03 \AA$, signaling a phase change.

electronic transition at $\mathrm{Ni}-\mathrm{O}$ is observed. The $\mathrm{Ni}$ moment initially decreases $\left(\Delta M / \Delta a \approx 1.6 \mu_{B} / \AA\right)$. When the Ni-O separation is lowered below the critical value of $a_{c} / 2 \equiv d_{c}^{\mathrm{Ni}-\mathrm{O}}=$ $2.03 \AA$, the rate of decrease of the Ni moment doubles.

At this same separation, the (magnetic) energy undergoes a dramatic change. The energy increases slowly and quadratically down to $4.06 \AA$. Below $a=4.06 \AA$, where Matsumoto and collaborators suggest a crossing of magnetic and nonmagnetic $\mathrm{Ni}$ ions, the energy difference rises steeply with a slope of $-300 \mathrm{meV} / \AA$. This electronic transition involves a reconfiguration of how orbital occupations change with volume.

This behavior is consistent with the critical Ni-O separation proposed by Matsumoto et al. They observed that most $\mathrm{NiO}_{2}$ compounds with only square planar $\mathrm{Ni}$ (no apical oxygens) are nonmagnetic, but if the lattice constant is pushed above $2.0 \AA$, the $\mathrm{Ni}$ ion becomes magnetic. For example, $\mathrm{Sr}_{2} \mathrm{NiO}_{2}(\mathrm{AgSe})_{2}$, with $a=4.094 \AA$, is magnetic; $\mathrm{BaNiO}_{2}$ and $\mathrm{LaNiO}_{2.5}$ are not. The significant aspect is that while our $\mathrm{Ni}$ ion is spin polarized, i.e., magnetism must be considered in the electronic structure, it forms a singlet and is not magnetic to most experimental probes.

\section{Electronic structure at the GGA level}

The band structure at the GGA level, which forms the basis for most beyond-GGA studies, is presented in Fig. 4 with the atom-projected density of states (PDOS). The $d_{z^{2}}$ band is degenerate with the $t_{2 g}$ bands, all with $0.5-1 \mathrm{eV}$ bandwidths. The Ni $d_{x^{2}-y^{2}}$ dispersion is $3 \mathrm{eV}$ (see the $\Gamma-M$ line) with

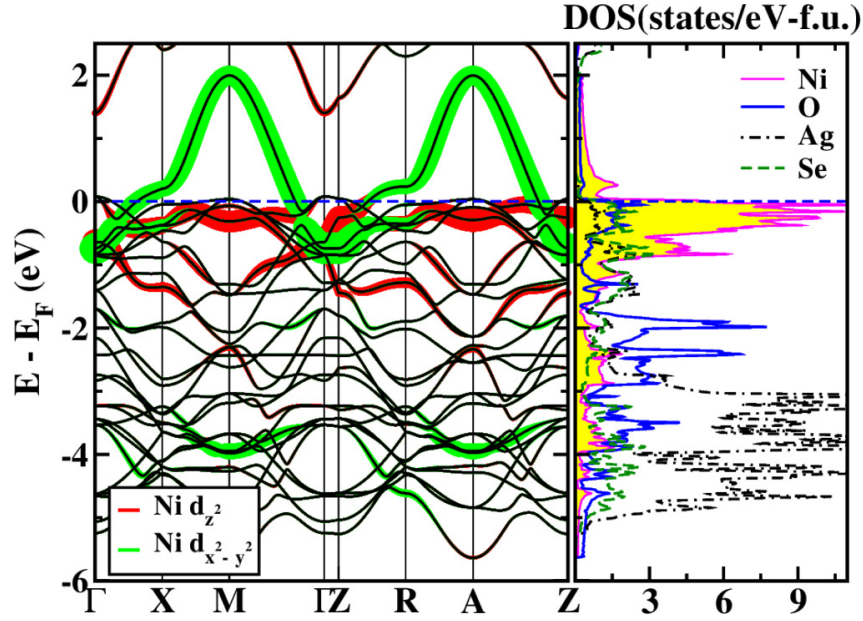

FIG. 4. Left: Reference GGA nonmagnetic band structure with highlighted fat bands of $\mathrm{Ni} d_{z^{2}}$ and $d_{x^{2}-y^{2}}$ in $\mathrm{Ba}_{2} \mathrm{NiO}_{2}(\mathrm{AgSe})_{2}$. Right: Corresponding atom-projected densities of states. Most of the narrow $d_{z^{2}}$ band lies just below the Fermi energy $E_{F}$. The mostly empty $d_{x^{2}-y^{2}}$ band has bandwidth $\sim 3 \mathrm{eV}$, corresponding to in-plane hopping $t \approx 0.4 \mathrm{eV}$.

the center of weight around $0.5 \mathrm{eV}$, giving an $e_{g}$ crystal-field splitting of $1-1.5 \mathrm{eV}$. The lowest conduction band at $\Gamma$ has $\mathrm{Se}$ $p_{z}$ and $\mathrm{Ag} s$ characters; this will be referred to as the $\mathrm{Se}-\mathrm{Ag}$ band and attains importance for electron doping, discussed below.

Dimensionality of the band structure is one central point of interest in nickelates as well as cuprates. $k_{z}$ dispersion along $\Gamma-Z$ of valence bands is small except for (1) the $\mathrm{Ni}$ $d_{z^{2}}$ band just below $E_{F}$, with a dispersion of roughly $0.5 \mathrm{eV}$, accommodated by hybridization with the Se $p$ orbitals, i.e., through the electronic polarization of the blocking layer, and (2) the Se-Ag conduction band, which shows an in-plane dispersion of $1 \mathrm{eV}$ and $k_{z}$ dispersion from $\Gamma$ of nearly $0.5 \mathrm{eV}$. The two $3 d$ holes are accommodated mostly in the $d_{x^{2}-y^{2}}$ orbitals with a minor amount in the small Fermi surfaces with mostly $d_{z^{2}}$ character. These differences are central factors in how the Coulomb repulsion $U$ will produce changes in the spectral positions.

\section{Correlation and spin polarization: The ODS}

The PDOS displayed in the top panel of Fig. 5 reveals the paradigm-shifting effect of $U$. The ODS state is obtained already for $U=1 \mathrm{eV}$, though not yet favored energetically. The gap opens for $U$ in the $4-5 \mathrm{eV}$ range, beyond which the singlet is increasingly strongly favored [see Figs. 2(a) and 2(b)]. The spin density shown in Fig. 1(b) is strongly anisotropic (maximally so), similar to that obtained previously [2] for $d^{9} \mathrm{LaNiO}_{2}$, where large $U$ drove the system toward an unexpected $d^{8}$ configuration. $U=6-8 \mathrm{eV}$ was judged to be too large for conducting $\mathrm{LaNiO}_{2}$, which is observed to be conducting and would have a more strongly screened (smaller) value of $U$ [29].

A comparison with the FM progression $(S=1)$ in the lower panel of the PDOS in Fig. 5 reveals few differences aside from the flipped $d_{z^{2}}$ spin. At $U=1 \mathrm{eV}$, the occupied (unoccupied) $d_{z^{2}}$ states are at $-2 \mathrm{eV}(+0.2 \mathrm{eV}$, slightly 

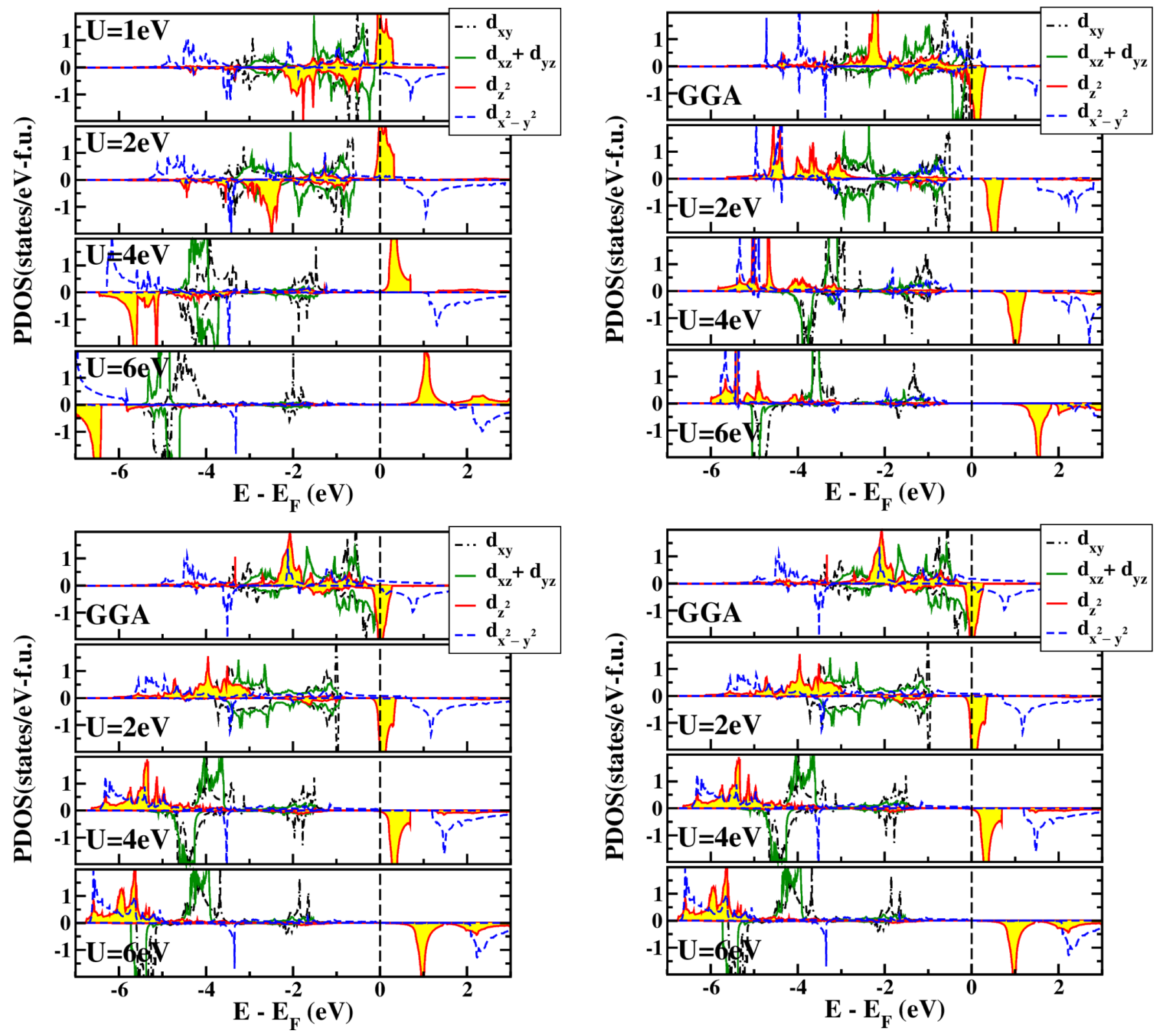

FIG. 5. Top: The Ni $d$ orbital-projected densities of states for the ODS state obtained from FPLO, vs strength of $U$. Bottom: The corresponding FM figure is shown for comparison; there is little difference except for the direction of the unoccupied $d_{z^{2}}$ spin. Including $U$ even at the $1 \mathrm{eV}$ level, the spin of the (partially) unfilled $d_{z^{2}}$ is reversed, leading to the ODS singlet state, still slightly conducting.

overlapping $E_{F}$ ). There is little difference in the positions and widths of most of the occupied $d$ bands. The noticeable difference is that the occupied $d_{z^{2}}$ band is $1.5 \mathrm{eV}$ lower for the FM case at $U=2 \mathrm{eV}$. A close look indicates that the $t_{2 g}$ PDOS is lower for FM than for ODS. At $U=1 \mathrm{eV}$ and above, the $d_{x^{2}-y^{2}}$ and $d_{z^{2}}$ "Mott gaps" increase proportional to $U$ with most of the separation being absorbed by increased binding of the occupied orbital.

\section{E. High spin: AFM vs FM alignment}

In Fig. 5 the orbital-projected density of states (PDOS) of the ODS was compared to that of the FM state, for

FIG. 6. Orbital-projected density of states vs $U$ for (upper panel) AFM alignment compared to (lower panel) FM alignment, both having high-spin Ni ions. For FM alignment, "up" is the direction of the $d_{x^{2}-y^{2}}$ spin and is plotted upward, and "down" spin is plotted downward. For AFM, only spin-up (spin-down) indicates majority (minority) of a given $\mathrm{Ni}$ atom.

$U=0-6 \mathrm{eV}$. In Fig. 6 we make the analogous comparison between the AFM and FM alignments of $\mathrm{Ni}(S=1)$ spins, which we find to be instructive. Bandwidths change, because majority states can hop only to minority states on the nearest $\mathrm{Ni}$ which have different energies; thus AFM bandwidths are narrower than FM as usual. Differences in the energy position of orbital occupations may or may not change and can be judged by this information.

Figure 6 shows the progression of the Ni orbital-projected DOS as $U$ is increased from zero to $6 \mathrm{eV}$, for AFM alignment (above) and FM alignment (below). Each of these corresponds to a high-spin Ni ion. The $d_{z^{2}}$ PDOS, which is of most interest, 


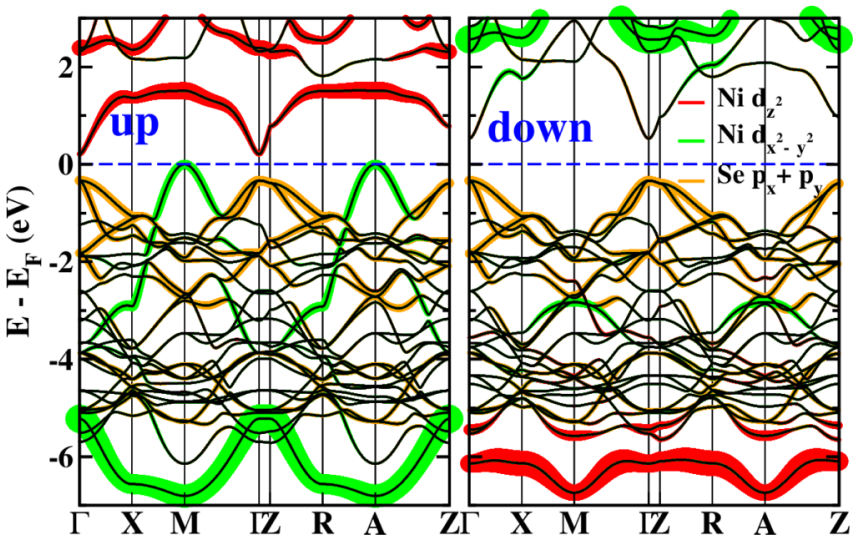

FIG. 7. Band structure of the ODS state at $U=7 \mathrm{eV}$ in GGA $+U$. The fat bands of Ni $d_{z^{2}}$, Ni $d_{x^{2}-y^{2}}$, and Se $p_{\pi}$ are highlighted. In the spin-up channel (left panel), the top of the valence bands occurs with a Se $4 p$ band at $\Gamma$ and a band at $M$ with some $d_{x^{2}-y^{2}}$ character. The valence band maximum for down spins (right panel) is a $\Gamma$-centered band of Se $p_{x}+p_{y}$ orbitals.

is emphasized in yellow. For AFM alignment, at $U=0$ holes reside primarily in $d_{x^{2}-y^{2}}$. The $d_{z^{2}}$ PDOS (occupied) is spread across $3 \mathrm{eV}$. At $U=2 \mathrm{eV}$, the gap opens, giving the full high-spin state of the $\mathrm{Ni}$ ion. By $U=4 \mathrm{eV}$, the (inactive) $t_{2 g}$ states stay in the -1 to $-2 \mathrm{eV}$ region. The occupied $e_{g}$ majority orbitals move to lower energy and become localized, so something nonlinear has occurred. $U=6 \mathrm{eV}$ is as $4 \mathrm{eV}$, except Mott splitting has increased. The nonlinearity is due to the $d$ states moving below the Se and $\mathrm{O} p$ bands, and is not of much consequence.

For FM alignment, at $U=0$ the state is metallic but is approximating the $S=1$ high-spin state. At $U=2 \mathrm{eV}$, tails of bands are still crossing $E_{F}$ but the $S=1$ configuration is emerging. Changes can be seen in the occupied $d$ region, as for AFM. Increasing to $U=4 \mathrm{eV}$, the spectrum is essentially gapped. $t_{2 g}$ states have moved to $-4 \mathrm{eV}$ and become localized. At $U=6 \mathrm{eV}$ the spin splitting of $t_{2 g}$ has increased, and the full $S=1$ state is attained. There is a $1 \mathrm{eV}$ difference in the position between the two minority holes. From Fig. 2(b) the AFM and FM energies are not very different.

This progression with $U$ seems about as expected: $U$ increases the separations between occupied and unoccupied orbitals, and pushes orbital occupations toward zero or unity. There is "nonlinearity" in the movement of occupied $d$ states between 2 and $4 \mathrm{eV}$, but this is probably due to moving out, then being pushed out, by the $\mathrm{O} 2 p$ or Se $4 p$ bands in the -3 to $-1 \mathrm{eV}$ region. The linear progression of the unoccupied orbitals, widening the gap as $U$ increases, is usual.

\section{ANALYSIS OF THE ODS}

\section{A. Electronic structure}

The band structure of the ODS state is shown in Fig. 7, with the corresponding PDOS of relevant orbitals pictured in Fig. 8. Both $e_{g}$ orbitals are split by roughly $U=7 \mathrm{eV}$ (the Mott-Hubbard gap between opposite spin orbitals), with unoccupied $d_{x^{2}-y^{2}}$ bands $1.5 \mathrm{eV}$ higher in energy due to the crystal field. A substantial effect of $U$ is to remove the $t_{2 g}$

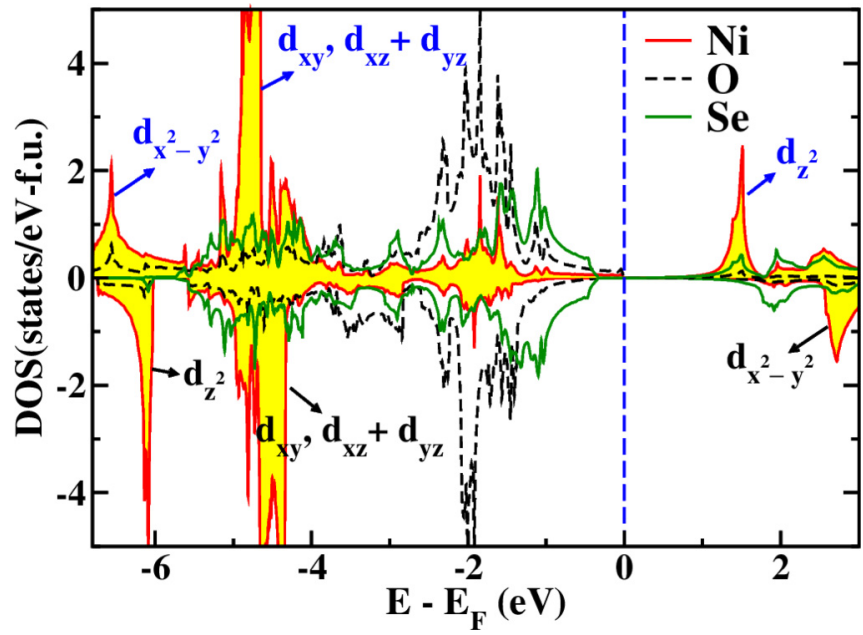

FIG. 8. Atom-projected densities of states (DOS) and Ni orbitalprojected DOS at $U=7 \mathrm{eV}$ in GGA $+U$ for the ODS state, with the unfilled spin-up $d_{z^{2}}$ and spin-down $d_{x^{2}-y^{2}}$ orbitals being evident. The $t_{2 g}$ orbitals have been lowered to -4 to $-5 \mathrm{eV}$ below the gap.

orbitals (both spins occupied) from just below the GGA Fermi level to -4 to $-5 \mathrm{eV}$ below the gap, where they can as usual be ignored. This displacement serves to leave the $\mathrm{Se} 4 p$ states at the bottom of the gap at $\Gamma$, with the $\mathrm{O} 2 p$ bands somewhat lower. Note: With zero net ODS spin, we have chosen that "up" is the direction of the $d_{x^{2}-y^{2}}$ spin, and "down" is of course the opposite.

The gap opening in both spin directions at $U_{c} \approx 5 \mathrm{eV}$ signals a metal-insulator transition and ensures that the net moment vanishes, giving the pure ODS state. This state violates Hund's first rule, signaling that other (intra-atomic and environmental) contributions to the energy are compensating. We propose that one factor arises from the differences in crystal-field splittings from neighboring divalent cations $(\mathrm{Ba}$ or $\mathrm{Sr}$ ) versus the tripositive ions in $d^{9} \mathrm{LaNiO}_{2}$ and $\mathrm{NdNiO}_{2}$. There are only minor differences between FPLO and WIEN2K, but what is important is that the transition to the ODS singlet and gap opening occurs in both codes, so it is a robust feature.

The unoccupied Ni $d_{z^{2}}$ hole band is, in first approximation, flat at $+1.3 \mathrm{eV}$. The dispersion that is seen in the Fig. 7 fat bands is due to rather strong mixing with the $\mathrm{Se}-\mathrm{Ag}$ conduction band that is dipping down at $\Gamma$. This hybridized band disperses about $1 \mathrm{eV}$ from $\Gamma$ to the zone edge $X$, and $0.5 \mathrm{eV}$ width along $k_{z}$. The gap increases to $0.5 \mathrm{eV}$ for $U=8 \mathrm{eV}$ (see Fig. 2), as occupancies attain essentially integer values. The spin density shown in Fig. 1(b) vividly illustrates the spin polarization that is a combination of $d_{z^{2}}$ and $d_{x^{2}-y^{2}}$ contributions of opposite spins, with $d_{x^{2}-y^{2}}$ orbitals mixed with oxygen $p_{\sigma}$ orbitals. We return in Sec. V to further implications of this ODS band structure.

The electron-hole asymmetry for $U>U_{c}$ is anomalous for a transition metal oxide, rendering the usual Mott insulator versus charge transfer insulator inapplicable. Oxygen $2 p$ states do hybridize with $d_{x^{2}-y^{2}}$ but not as strongly as conventionally, since they lie $2-4 \mathrm{eV}$ below the gap. Hole states (see Fig. 7) are instead Se $4 p$ states in the down-spin bands that are highly itinerant. $\mathrm{O} 2 p$ bands are somewhat more tightly bound. 
Electron states also present different behavior: Electrons initially go into the three-dimensional (3D) dispersive band at $\Gamma$ of the itinerant $\mathrm{Se}-\mathrm{Ag} 4 s$ band mixed with up-spin $d_{z^{2}}$ character. There is a $0.2 \mathrm{eV}$ exchange splitting, and the downspin $\mathrm{Se}-\mathrm{Ag}$ band does not mix with other bands. At a still small concentration electrons begin to occupy this spin-down strongly itinerant band. Significant doping levels will therefore produce up-carriers in the $\mathrm{NiO}_{2}$ layer and down-carriers in the AgSe layer, with associated spin polarization. The carriers can be expected to disturb the singlet, likely enabling further moments to emerge. A serious study of doping would require a separate study.

\section{B. Orbital occupations and charges}

Variations of spin-resolved $\mathrm{Ni}$ occupancies with $U$ are displayed in Fig. 9, comparing ODS with FM alignment. The occupation numbers $n_{m}$ of the active $e_{g}$ orbitals $m$ (top panels) show similar behavior, especially in the physically relevant region $U>3$, except that $d_{z^{2}}$ is flipped in spin in the ODS state. At the more detailed level, the hole occupation numbers for $S=1$ are constant for $U>3 \mathrm{eV}$, while for the singlet $S=0$ there is a small but continuing increase by $0.02-0.04$ up to $U=9 \mathrm{eV}$. Somewhat unexpected is that it is $d_{x^{2}-y^{2}}$ that changes most, but is understandable because that dispersive band crosses $E_{F}$ at small $U$ and $U=5 \mathrm{eV}$ is required to completely empty it.

The atomic orbital basis of FPLO provides atomic Mulliken charges (MCs), in which contributions to the density from overlapping orbitals of neighboring atoms are divided equally between the atoms; the sum of MCs equals the total charge. The change of MCs with $U$, shown in Fig. 9 (middle and bottom panels), provides a complementary view of correlation effects, since they reflect changes in bonding (viz., relative orbital energies) as well as actual charge rearrangement. The atomic MCs (neutral atom values) for $\mathrm{Ba}, \mathrm{Ni}, \mathrm{O}, \mathrm{Ag}$, and $\mathrm{Se}$ are 54.6 (56), 27.2 (28), 9.2 (8), 46.9 (47), and 34.7 (34), respectively. These are quite reasonable values for MCs, and are not expected to correspond closely to the formal charges. All do differ from the neutral atom value in the "right direction," and by roughly half of the formal valence.

We focus on the $\mathrm{Ni} \mathrm{MC}$ variation (any change on $\mathrm{Ni}$ must go somewhere in the Mulliken decomposition, and that somewhere is the atoms $\mathrm{O}$ and $\mathrm{Se}$ that hybridize with $\mathrm{Ni}$ ). The changes in total Ni Mulliken ionicity with increasing $U$ (lower two panels) are similar for ODS and FM states, saturating for Ni $S=0$ around $U=6$ at a value of -0.2 while for $S=1$ there is less tendency toward saturation. The spin-resolved $\mathrm{MC}$ ionicities (middle panels) differ qualitatively between FM and ODS states. For ODS $S=0$ the change in down MC is a small fraction of that of the up, which is in the -0.18 range. For FM $S=1$ the down MC charge becomes twice as large, while that of up is roughly half as large and of opposite sign. At $U=1 \mathrm{eV}$, the Ni moment is $-0.18 \mu_{B}$, but the net moment is zero. With increasing $U$, the down-spin charge moves to the other spin and other ions. Above $U \approx 4 \mathrm{eV}$, the magnitude of the Ni moment vanishes, entering a true ODS phase. We are not aware of nickelate or cuprate values of MCs to compare with.
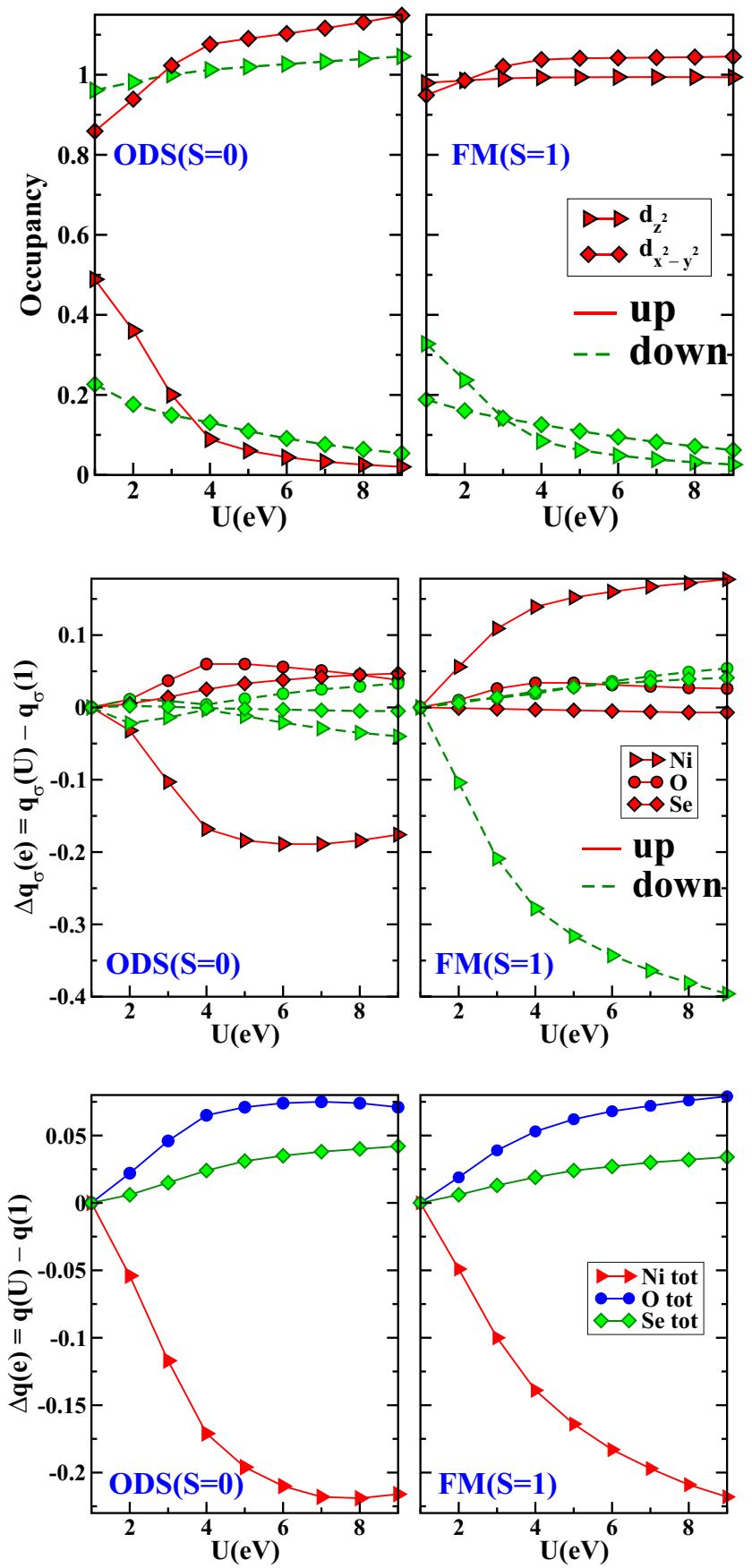

FIG. 9. Variations of measures of charge of $S=0$ (singlet) and $S=1$ (FM) states vs $U$. Top: Occupation matrix elements $n_{d_{j}}$ of the active Ni orbitals. Middle (bottom): FPLO Mulliken charges of atoms as labeled for $S=0(S=1)$, compared to their values at $U=1$ (because a singlet state was not obtained at $U=0$ ).

\section{KONDO SIEVE SPIN MODEL FOR BNOAS}

\section{A. The model}

The insulating ground state ODS that is discussed above provides a system in which the elementary excitations are spin waves. We suggest here a minimal model of the spin system. Let the $d_{x^{2}-y^{2}}$ and $d_{z^{2}}$ spin operators be denoted by the Pauli matrices $\vec{\sigma}$ and $\vec{\tau}$, respectively. Factors of $\frac{1}{2}$ will be 
folded into the constants. The minimal spin Hamiltonian for the insulating state contains three parameters:

$$
\begin{aligned}
H & =J \sum_{\langle i, j\rangle} \vec{\sigma}_{i} \cdot \vec{\sigma}_{j}+K \sum_{i} \vec{\sigma}_{i} \cdot \vec{\tau}_{i}+J_{z} \sum_{[i, j]} \vec{\tau}_{i} \cdot \vec{\tau}_{j}-\sum_{i} \vec{S}_{i} \cdot \vec{B} \\
& =H_{\mathrm{KS}}+J_{z} \sum_{[i, j]} \vec{\tau}_{i} \cdot \vec{\tau}_{j}-\sum_{i} \vec{S}_{i} \cdot \vec{B} .
\end{aligned}
$$

$J \equiv J_{\sigma}$ is the positive in-plane near-neighbor $(\langle i, j\rangle)$ superexchange constant between $d_{x^{2}-y^{2}} \sigma$ spins, and $K$ is the on-site spin coupling between $\vec{\sigma}$ and "Kondo-like" $\vec{\tau}$ spins, with contributions from Hund's coupling, Hubbard $U$ correlation, and environmental influences. The on-site singlet-triplet splitting is $2 K$, and the sign of interest here (making it Kondo-like) being positive $K . J_{z}$ is the (spacer layer assisted) coupling between $\tau$-spin neighbors $[i, j]$ along $\hat{z}$, and the total on-site spin is $\vec{S}_{i}=\vec{\sigma}_{i}+\vec{\tau}_{i}$ couples to the magnetic field $\vec{B}$. For the discussion in this section we set units such that $J=1$.

$H_{\mathrm{KS}}$, which we refer to as the Kondo sieve model, is an extension to 2D of the Kondo necklace model introduced by Doniach [30]. It was derived from the 1D Kondo Hamiltonian by applying the Luther-Pelcher transformation [31] from 1D spinless itinerant particles to on-site spins. Quantum Monte Carlo calculations indicate that the 1D Kondo necklace does not display magnetic order at any finite value of the exchange coupling constant [32]. The extension to 2D by Brenig [33] has been known (somewhat contradictorily) as the 2D Kondo necklace. This two spins per site model can also be viewed as the large Hubbard $U$ regime of the Kondo-Hubbard model at half filling [34]. We picture BNOAS, in first approximation, as a realization of the Kondo sieve model, then with small and mostly frustrated interlayer coupling that may become important in the emergence of ordered phases.

Quite a bit is known from Brenig about the Kondo sieve model. For $J=0, H_{\mathrm{KS}}$ consists of isolated singlets; for $K=0$ it is the exhaustively studied spin-half 2D AFM Heisenberg model, which does not order at finite $T$ (Mermin-Wagner theorem). Using stochastic series expansion methods, Brenig found that weak AFM order exists for $H_{\mathrm{KS}}$ at low $T=0.05$ up to a quantum critical point (QCP) $[33,34] K_{c}=1.4$. Below $K_{c}$ the $\tau$-projected order parameter is around $35 \%$ larger than that of the neighbor-coupled $\sigma\left(d_{x^{2}-y^{2}}\right)$ spins.

The ordering reveals that the Kondo spin promotes longrange order even in 2D, and becomes a central influence even for a small Kondo coupling, reflecting apparent smaller quantum fluctuations than the $\sigma$ spin. The uniform susceptibility decreases with increasing $K$, vanishing at $K_{c}$ above which the singlets control the physics. Site dilution of the "Kondo" moments, as by nonstoichiometry or doping, results in a structure in both $\chi\left(Q_{\mathrm{AFM}}, T\right)$ and $S\left(Q_{\mathrm{AFM}}, T\right)$ around $T=0.1$ or below, depending on concentration. The QCP vanishes into a crossover, i.e., weak AFM order extends to larger $K$, for which an order-by-disorder mechanism was proposed [34]. These results will also impact the doping experiments proposed below.

\section{B. Relation to $\mathrm{Ba}_{2} \mathrm{NiO}_{2}(\mathrm{AgSe})_{2}$}

Due to the body-centered stacking of $\mathrm{NiO}_{2}$ layers, both the $\tau$ and also much smaller $\sigma$ couplings between AFM layers will be frustrated. The $0.5 \mathrm{eV} k_{z}$ dispersion of the $d_{z^{2}}$ band imbues importance to this coupling, since it is the largest coupling available to enable 3D magnetic correlations and potential long-range 3D order. An in-plane term $\sum_{\langle i, j\rangle} \vec{\tau}_{i} \cdot \vec{\tau}_{j}$ and interlayer coupling $\sum_{[i, j]} \vec{\sigma}_{i} \cdot \vec{\sigma}_{j}$ are symmetry allowed, but both should be small.

Of special interest, insightful for the large $K$ that our calculations indicate, is that the on-site $(i)$ term can be diagonalized in total spin $\vec{S}_{i}=\vec{\sigma}_{i}+\vec{\tau}_{i}$ space, with singlet $S_{i}=0$ and triplet $S_{i}=1$ sectors. For the isolated ion $K$ is negative, giving the familiar high-spin Hund's coupling. In BNOAS we find instead from total energy results that the low-spin ODS configuration is favored (rather strongly) for physical values of $U$ [see Fig. 2(b)]. The nonmagnetic ${ }^{1} A$ state with two holes in the $d_{x^{2}-y^{2}}$ orbital is highly disfavored and is not included in the Kondo sieve model.

Given that the low-spin ODS state is favored in BNOAS, in zeroth approximation configurations are confined to the ODS sector $S_{i}=0$ for all sites $i$. Then the second term in $H$ becomes diagonal and the fourth term in the Hamiltonian vanishes (and also hence the linear susceptibility as in experiment), and $\vec{\tau}_{i}=\vec{S}_{i}-\vec{\sigma}_{i}$ can be eliminated to give (up to a constant)

$$
H \rightarrow \bar{H}_{\text {singlet }}=J \sum_{\langle i, j\rangle} \vec{\sigma}_{i} \cdot \vec{\sigma}_{j}+J_{z} \sum_{[i, j]} \vec{\sigma}_{i} \cdot \vec{\sigma}_{j} .
$$

The in-plane superexchange $J$ is ubiquitously calculated to be positive and large in nickelates, so the first term is the one that is commonly applied to the AFM insulating phase of cuprates. Since the $\sigma$ spins are antialigned (or at least strongly correlated), the body centering of the BNOAS structure determines that interlayer $\vec{\tau}_{i} \cdot \vec{\tau}_{j}$-here transformed to $\vec{\sigma}_{i} \cdot \vec{\sigma}_{j}$-coupling is frustrated.

The result from the Kondo sieve model without considering further symmetry breaking (such as single-ion anisotropy or structural distortion) is that the $\sigma$ spins are strongly correlated in plane and become ordered below $K_{c} / J=1.4$, with experimental properties becoming nonstandard for a magnetically ordered system. The substantial Kondo coupling indicates that weak AFM should be considered in the interpretation of magnetization data. Ordering across the layers due to additional interactions may be a lower-temperature possibility that is left for further work. For comparison with experimental data, the sensitivity of the QCP to defects should be kept in mind, since the synthesis methods of this class of compounds have been found to allow the incorporation of impurities and produce some minority phases [35].

\section{DISCUSSION}

Our results suggest that the non-Curie-Weiss form and the peculiar structure in $\chi(T)$ of $\mathrm{Ba}_{2} \mathrm{NiO}_{2}(\mathrm{AgSe})_{2}$ are couched in the formation of a $\mathrm{Ni}$ on-site "off-diagonal singlet" in which both $e_{g}$ orbitals are singly occupied but with Kondo-like oppositely spin-directed singlets. This singlet forming tendency was obtained previously [2] for infinite-layer $\mathrm{LaNiO}_{2}$ at large interaction $U$-very strange for a supposed $d^{9}$ metal-but seemed not to be reflected in the measured properties.

This singlet with intersite coupling of only one of the components leads us to propose that a bare-bones model of $\mathrm{Ba}_{2} \mathrm{NiO}_{2}(\mathrm{AgSe})_{2}$ is given realistically by the Kondo sieve model (2D Kondo necklace), for which some essential characteristics are known from quantum Monte Carlo studies. 
Among the numerous theoretical viewpoints of $\mathrm{NdNiO}_{2}$, the undoped parent of the newly discovered superconducting nickelate, Zhang et al. have suggested that Kondo singlets play a part in the underlying electron structure [36]. We propose that BNOAS may comprise a realization of Kondo sieve physics, and that it may provide important insight into layered nickelate physics which now includes superconductivity when appropriately doped $[6,7]$. We now turn to consideration of the peculiar behavior of $\chi(T)$ reported from experiment.

\section{A. Scenario No. 1: Integrity of the singlet}

Above the characteristic temperature $T_{m}=130 \mathrm{~K}$ of BNOAS, the $S=0$ singlets are magnetically inert, which naturally accounts for the lack of a Curie-Weiss term. The internal structure presumably is correlated due to antiferromagnetic $d_{x^{2}-y^{2}}$ coupling, but contributing a bit to $\chi(T)$ due to mixing of the $S=1$ triplet, consistent with experiment. In the language of the Kondo sieve model, the ordering below $T_{m}$ means that $K<K_{c}=1.4 J$, i.e., below the QCP where ordering occurs. As long-range ordering of the $d_{x^{2}-y^{2}}$ internal structure emerges at and below $T_{m}$, magnetic behavior emerges, apparently requiring involvement of the $S=1$ sector, only to return (in zero-field-cooled data) to invisibility in $\chi$ in the ordered state. This long-range correlation and ordering of weak moments (including a small orbital moment, which we calculate to be $0.16 \mu_{B}$ parallel to the $d_{x^{2}-y^{2}}$ moment) should be detectable, though possibly challengingly so, by neutron diffraction, by utilizing the magnetic (spin+orbital) structure factor that contributes to the magnetic density correlation function. Muon spin resonance is also a particularly sensitive method to detect magnetic order, though less so for magnetic correlations.

Probably more telling is that the susceptibility will be distinct from that of truly nonmagnetic ions, with some $T$ dependence arising from a van Vleck contribution from the proximity in energy of the $S=1$ states. The field-cooled susceptibility below $T_{m}$ may arise from the domain structure in which $S=1$ moments at the domain boundaries come into play. To repeat from above: extrinsic phases may interfere in the data with intrinsic behavior, complicating interpretation.

\section{B. Scenario No. 2: Quenching of the singlet}

The preceding discussion has assumed the integrity of the quantum ODS singlet. This is textbook behavior as long as the two spins remain in a coherent singlet, which conventionally assumes degenerate orbitals and negligible coupling to the environment. Neither of these conditions applies strictly to BNOAS. At elevated temperature these "perturbations" may average out so the singlet survives, but may become more influential as temperature is lowered. Similarly to the quenching of atomic orbital angular momentum by environmental effects (nonspherical potential), the ODS may revert to the nonquantum pair of individual but coupled $\vec{\sigma}$ and $\vec{\tau}$ spins (as described by DFT). Coupling then will strictly antialign the individual orbital spins, which can then separately respond also to an applied magnetic field, and have separate (and unequal) orbital contributions and provide a distinct susceptibility though probably still small. The magnetic transition at $T_{m}$ could, in this scenario, correspond to the quenching of the quantum singlet, with consequences that may not have been studied previously.

\section{Doping possibilities}

Electron doping, which would drive the $\mathrm{Ni}$ ion toward the $d^{9}$ configuration where high-temperature superconductivity (HTS) occurs in cuprates, is of primary interest. Figure 7 indicates an unusual band structure for such a strongly layered nickelate discussed in Sec. IIID, with the characters of the bands bordering the gap described in Sec. IV A. At very low doping carriers go into a band that disperses out of plane as much as in plane: spheroidal Fermi surfaces. With increased doping the spheres will join along the short $k_{z}$ dimension of the zone, leaving roughly cylindrical Fermi surfaces. At higher dopings continued conduction can be expected, arising from very different spin-up and spin-down contributions as discussed in Sec. IV A: spin-up electrons in a largely $d_{z^{2}}$ band, spin-down electrons in the AgSe layer.

The most obvious candidate is $\mathrm{La}^{3+}$ substitution for $\mathrm{Ba}^{2+}$, which gave the initial HTS (La, Ba) ${ }_{2} \mathrm{CuO}_{4}$. Such $\mathrm{La} \leftrightarrow \mathrm{Ba}$ substitutions are common, and with their electropositivities they do little other than change the carrier concentration and to affect the volume slightly. Similarly, $\mathrm{Ag}^{+}$replaced by $\mathrm{Cd}^{2+}$ might have similar effects.

Substituting on the anion sites is the other possibility. Replacement of $\mathrm{O}^{2-}$ by $\mathrm{Cl}^{-}$can achieve similar effects, with the difference that bonding with $\mathrm{Ni}$ is affected to some degree. $\mathrm{Se}^{2-}$ replacement by $\mathrm{Br}^{-}$is less commonly tried (or at least achieved). Finally, one can consider $\mathrm{Ni}^{2+}$ replaced by $\mathrm{Co}$ or $\mathrm{Cu}$. These might assume the $2+$ charge state, which would give no doping but disturb the singlet character. Chemical behavior at high pressure may determine which type of replacement is most likely to retain the crystal structure and provide electron doping.

Hole doping seems less compelling. The states are primarily $\mathrm{Se} 4 p$ in character and essentially spin degenerate. This itinerant charge in the AgSe layer does not change the $\mathrm{Ni}$ valence, and does not even screen the $\mathrm{Ni}$ ion, i.e., does not change $U$ on $\mathrm{Ni}$ nor the gap in the $\mathrm{NiO}_{2}$ layer. In clean crystalline samples it might produce a high-mobility $2 \mathrm{D}$ electron gas, with its well-studied properties. Having this 2D hole gas interlayered with (ordered) ODSs would, however, be a novel system to study.

\section{Analogous materials}

Related behavior was observed in DFT $+U$ studies of $\mathrm{MnO}$ under pressure, which both experiment and theory identify as a high-spin to low-spin (but not zero-spin) transition [37] in the vicinity of an insulator-metal transition. In DFT $+U$ calculations [28], the low-pressure $S=\frac{5}{2}$ Mn ion first undergoes, under pressure, an insulator-to-insulator transition in which two Mn $3 d$ orbitals flip spin direction while each orbital remains fully spin polarized, i.e., an $S=\frac{5}{2} \rightarrow S=\frac{1}{2}$ spin collapse without a change in orbital occupation. This spin collapse amounts to an even more drastic violation of Hund's first rule than in BNOAS that is discussed here. The origin of the energy gain was traced to the persisting strong (maximal) anisotropy in spin and charge, and to a change in $p d \sigma$ hybridization. In that system $\mathrm{MnO}$ was also in 
proximity to a critical metal-oxygen separation. Both of these characteristics (anisotropy and a critical bond length) are also apparent in BNOAS.

A prime interest in BNOAS will be how it may inform the electronic structure and magnetic behavior in superconducting $(\mathrm{Nd}, \mathrm{Sr}) \mathrm{NiO}_{2}$. As mentioned, the ODS tendency was seen 15 years ago in $\mathrm{LaNiO}_{2}$, which is commonly used as a stand-in for $\mathrm{NdNiO}_{2}$ in correlated electron calculations. It should be mentioned that others have not reported obtaining this singlet tendency in $\mathrm{LaNiO}_{2}$. However, in BNOAS we obtain it with two different codes and $\mathrm{GGA}+U$ implementations. We obtain the magnetic states others report but they have higher energies (for physical values of $U$ ). There are still things to be learned about the peculiar behavior of the $d_{z^{2}}$ orbital in infinite-layer nickelates. It is of considerable interest to try to electron-dope BNOAS toward the $d^{9-\delta}$ regime where superconductivity emerges in $(\mathrm{Nd}, \mathrm{Sr}) \mathrm{NiO}_{2}$.

Another related system is $\mathrm{Ba}_{2} \mathrm{CuO}_{3.2}$, which superconducts at $73 \mathrm{~K} \mathrm{[38]} \mathrm{after} \mathrm{having} \mathrm{been} \mathrm{synthesized} \mathrm{at} 18 \mathrm{GPa}$ and $1000{ }^{\circ} \mathrm{C}$. Although not described as such, this compound is an infinite-layer cuprate that is hole doped with 0.2 apical oxygen atoms per copper, making it formally somewhat overdoped. The (average) apical $\mathrm{Cu}-\mathrm{O}$ distance is small at $1.86 \AA$, whereas the in-plane $\mathrm{Cu}-\mathrm{O}$ separation is $2.00 \AA$, larger than other superconducting cuprates. The interpretation of spectral data suggested that the crystal field has exchanged the positions of the $d_{z^{2}}$ and $d_{x^{2}-y^{2}}$ levels, which would provide another paradigm for cuprate superconductivity. The relationship of this material to BNOAS suggests the usefulness of the further study of both systems.

While BNOAS is not simple to synthesize due to the need for high pressure, it is nevertheless available (after synthesis) to experimental probes that are not available to $\mathrm{MnO}$ at $100 \mathrm{GPa}$ pressure. We encourage further synthesis and study of BNOAS by thermodynamic and spectroscopic (electron, neutron, and muon) probes to illuminate the peculiar magnetic behavior observed in this infinite-layer nickelate.

\section{SUMMARY}

Our density functional theory with correlation effects study of $\mathrm{Ba}_{2} \mathrm{NiO}_{2}(\mathrm{AgSe})_{2}$ has uncovered the likelihood that an off-diagonal singlet arises in the subspace of the two $e_{g}$ holes (or electrons) in its $d^{8}$ ionic configuration. This "low-spin" singlet, however, has orbital polarization, i.e., orbital texture, with one orbital's spin being coupled to neighbors while the other is not. Moreover, a critical Ni-O separation of $2.03 \AA$ has been obtained, which seems to be associated with the distinction between low-spin and high-spin $\mathrm{Ni} d^{8}$ ions in square planar coordination, occurring at the same Ni-O distance. Two scenarios have been presented, either of which might account for the peculiar measured susceptibility: a magnetic transition at $T_{m}=130 \mathrm{~K}$ but nonmagnetic behavior above $T_{m}$.

\section{ACKNOWLEDGMENTS}

We acknowledge assistance from Young-Joon Song and Mi-Young Choi in the early stages of this work. The spin model aspects of this paper have benefited from feedback and references from R. T. Scalettar and comments from R. R. P. Singh. We appreciate extensive comments on the manuscript from M. D. Johannes and thank A. B. Shick for the use of his code that calculates the anisotropic $U$ and $J$ matrices reported in the Appendix. H.-S.J. and K.-W.L. were supported by National Research Foundation of Korea Grant No. NRF2019R1A2C1009588. W.E.P. was supported by National Science Foundation Grant No. DMR 1607139.

\section{APPENDIX: ANISOTROPY OF $U_{m, m^{\prime}}$ AND $J_{m \cdot m^{\prime}}$}

The $U$ and $J$ matrix elements introduce orbital-occupation differences into the potentials and energies of the $\mathrm{DFT}+U$ results that are then included self-consistently. We provide these matrix elements for the study of some of these differences that arise. The importance of on-site anisotropy has been noted previously by several groups. The study of the "flipped spin" phenomenon in $\mathrm{MnO}$ under pressure by Kasinathan et al. [28], mentioned in the main text, revealed that the anisotropy of Coulomb repulsion and exchange parameters is deeply involved in the energetics causing that flipped spin from $S=\frac{5}{2}$ to $S=\frac{1}{2}$ while all $3 d$ orbitals of Mn remain fully spin polarized.

The situation we find in BNOAS is strongly analogous, so we provide below the matrix elements that enter the DFT $+U$ calculations. We provide the matrix elements for $U=5 \mathrm{eV}$, $J=0.7 \mathrm{eV}$. The Slater integrals $(\mathrm{eV})$ are $F_{0}=5.00, F_{2}=$ $6.03, F_{4}=3.77$, and the corresponding orbital-resolved matrix elements that occur in the $\mathrm{DFT}+U$ functional (in $\mathrm{eV}$ ) are given just below. This connection between the Slater integral and $U$ and $J$ is important to understand.

Features to notice are (1) $U_{m m}=J_{m m}$ : Self-exchange equals self-interaction, which the functional attempts to account for, and (2) the anisotropies in $U_{m m^{\prime}}$ and $J_{m m^{\prime}}$ are both proportional to $J$. $J$ describes both the anisotropy of the Coulomb repulsion and the anisotropy of the exchange interaction. Setting $J$ equal to zero results in a $U$ matrix with all elements equal to $U$, and of course a diagonal $J$ matrix with diagonal elements equal to $U-$ no anisotropy whatsoever.

The matrices are given below in the complex $\ell, m$ representation of $3 d$ orbitals. In this representation the matrices are symmetric around both diagonals.

The $U$ matrix:

\begin{tabular}{lccccc}
$m_{l}$ & -2 & -1 & 0 & +1 & +2 \\
\hline-2 & 5.501 & 4.720 & 4.559 & 4.720 & 5.501 \\
-1 & 4.720 & 5.260 & 5.041 & 5.260 & 4.720 \\
0 & 4.559 & 5.041 & 5.800 & 5.041 & 4.559 \\
+1 & 4.720 & 5.260 & 5.041 & 5.260 & 4.710 \\
+2 & 5.501 & 4.720 & 4.559 & 4.720 & 5.501 \\
\hline
\end{tabular}

The $J$ matrix:

\begin{tabular}{lccccc}
$m_{l}$ & -2 & -1 & 0 & +1 & +2 \\
\hline-2 & 5.501 & 0.781 & 0.621 & 0.299 & 0.598 \\
-1 & 0.781 & 5.260 & 0.380 & 1.080 & 0.299 \\
0 & 0.621 & 0.380 & 5.800 & 0.380 & 0.621 \\
+1 & 0.299 & 1.080 & 0.380 & 5.260 & 0.781 \\
+2 & 0.598 & 0.299 & 0.621 & 0.781 & 5.501 \\
\hline
\end{tabular}


Suppose all $3 d$ orbitals are fully occupied or empty $n_{d_{i}}=$ 1 or 0 . FM $S=1$ and singlet $S=0$ have the same two $e_{g}$ orbitals occupied (and same two unoccupied, with opposite spin). The $+U$ term is spin-blind, so there is no difference. In the $J$ matrix, the parallel-spin $S=1$ term has $J_{x^{2}-y^{2}, z^{2}}$, compared to zero for $S=0$ (the $J$ term is parallel spin only). Actually, one should really count all the pairs of occupied states instead of unoccupied states. Anyway, this term favors the high-spin, not the singlet, state (by $0.62 \mathrm{eV}$ ), as the simple Hund's rule suggests but gains more from the off-diagonal repulsion $U$. Kasinathan et al. provide an analysis of how the Hund's exchange energy can be overcome, with the above anisotropy being a central player.
[1] J. Chaloupka and G. Khaliullin, Orbital Order and Possible Superconductivity in $\mathrm{LaNiO}_{3} / \mathrm{LaMO}_{3}$ Superlattices, Phys. Rev. Lett. 100, 016404 (2008)

[2] K.-W. Lee and W. E. Pickett, Infinite-layer $\mathrm{LaNiO}_{2}: \mathrm{Ni}^{1+}$ is not $\mathrm{Cu}^{2+}$, Phys. Rev. B 70, 165109 (2004).

[3] A. S. Botana, V. Pardo, and M. R. Norman, Electron doped layered nickelates: Spanning the phase diagram of the cuprates, Phys. Rev. Mater. 1, 021801(R) (2017).

[4] J. Zhang, A. S. Botana, J. W. Freeland, D. Phelan, H. Zheng, V. Pardo, M. R. Norman, and J. F. Mitchell, Large orbital polarization in a metallic square-planar nickelate, Nat. Phys. 13, 864 (2017)

[5] A. S. Botana and M. R. Norman, Similarities and Differences between $\mathrm{LaNiO}_{2}$ and $\mathrm{CaCuO}_{2}$ and Implications for Superconductivity, Phys. Rev. X 10, 011024 (2020).

[6] D. Li, K. Lee, B. Y. Wang, M. Osada, S. Crossley, H. R. Lee, Y. Cui, Y. Hikita, and H. Y. Hwang, Superconductivity in an infinite-layer nickelate, Nature (London) 572, 624 (2019).

[7] S. Zeng, C. S. Tang, X. Yin, C. Li, Z. Huang, J. Hu, W. Liu, G. J. Omar, H. Jani, Z. S. Lim, K. Han, D. Wan, P. Yang, A. T. S. Wee, and A. Ariando, Phase diagram and superconducting dome of infinite-layer $\mathrm{Nd}_{1-x} \mathrm{Sr}_{x} \mathrm{NiO}_{2}$ thin films, arXiv:2004.11281.

[8] Y. Matsumoto, T. Yamamoto, K. Nakano, H. Takatsu, T. Murakami, K. Hongo, R. Maezono, H. Ogino, D. Song, C. M. Brown, C. Tassel, and H. Kageyama, High-pressure synthesis $\mathrm{A}_{2} \mathrm{NiO}_{2} \mathrm{Ag}_{2} \mathrm{Se}_{2}(\mathrm{~A}=\mathrm{Sr}, \mathrm{Ba})$ with a high-spin $\mathrm{Ni}^{2+}$ in squareplanar coordination, Angew. Chem. Int. Ed. 58, 756 (2019), see also their Supplemental Material.

[9] M. A. Hayward, Synthesis and magnetism of extended solids containing transition-metal cations in square-planar, $\mathrm{MO}_{4}$ coordination sites, Inorg. Chem. 58, 11961 (2019).

[10] M. Matsuda, K. Katsumata, A. Zheludev, S. M. Shapiro, and G. Shirane, Neutron scattering study in $\mathrm{BaNiO}_{2}$, J. Phys. Chem. Solids 60, 1121 (1999).

[11] M.-Y. Choi, K.-W. Lee, and W. E. Pickett, Role of $4 f$ states in infinite-layer $\mathrm{NdNiO}_{2}$, Phys. Rev. B 101, 020503(R) (2020).

[12] M. Crespin, P. Levitz, and L. Gatineau, Reduced forms of $\mathrm{LaNiO}_{3}$ perovskite. Part 1.-Evidence for new phases: $\mathrm{La}_{2} \mathrm{Ni}_{2} \mathrm{O}_{5}$ and $\mathrm{LaNiO}_{2}$, J. Chem. Soc., Faraday Trans. 2 79, 1181 (1983).

[13] M. A. Hayward, M. A. Green, M. J. Rosseinsky, and J. Sloan, Sodium Hydride as a powerful reducing agent for topotactic oxide de-intercalation: Synthesis and characterization of the nickel(I) oxide $\mathrm{LaNiO}_{2}$, J. Am. Chem. Soc. 121, 8843 (1999).

[14] M. A. Hayward and M. J. Rosseinsky, Synthesis of the infinite layer $\mathrm{Ni}(\mathrm{I})$ phase $\mathrm{NdNiO}_{2+x}$ by low temperature reduction of $\mathrm{NdNiO}_{3}$ with sodium hydride, Solid State Sci. 5, 839 (2003).
[15] K. Koepernik and H. Eschrig, Full-potential nonorthogonal local-orbital minimum-basis band-structure scheme, Phys. Rev. B 59, 1743 (1999).

[16] K. Schwarz and P. Blaha, Solid state calculations using WIEN2k, Comput. Mater. Sci. 28, 259 (2003).

[17] The orbitals retained in the band problem are $\mathrm{Ba}$ $5 s 5 p 6 s(7 s 5 d 6 p)$, Ni $3 s 3 p 3 d 4 s(4 p 4 d 5 s), \mathrm{O} 2 s 2 p(3 s 3 p 3 d), \mathrm{Ag}$ $4 s 4 p 4 d 5 s(5 p 5 d 6 s)$, and Se $3 s 3 p 3 d 4 s 4 p(4 d 5 s 5 p)$. The orbitals in parentheses indicate semicore or polarization orbitals.

[18] J. P. Perdew, K. Burke, and M. Ernzerhof, Generalized Gradient Approximation Made Simple, Phys. Rev. Lett. 77, 3865 (1996).

[19] E. R. Ylvisaker, W. E. Pickett, and K. Koepernik, Anisotropy and magnetism in the LSDA+U method, Phys. Rev. B 79, 035103 (2009).

[20] V. I. Anisimov, I. V. Solovyev, M. A. Korotin, M. T. Czyzyk, and G. A. Sawatzky, Density-functional theory and NiO photoemission spectra, Phys. Rev. B 48, 16929 (1993).

[21] J. Karp, A. S. Botana, M. R. Norman, H. Park, M. Zingl, and A. Millis, Many-Body Electronic Structure of $\mathrm{NdNiO}_{2}$ and $\mathrm{CaCuO}_{2}$, Phys. Rev. X 10, 021061 (2020).

[22] W. E. Pickett, S. C. Erwin, and E. C. Ethridge, Reformulation of the LDA+U method for a local-orbital basis, Phys. Rev. B 58, 1201 (1998).

[23] M. Cococcioni and S. de Gironcoli, Linear response approach to the calculation of the effective interaction parameters in the LDA+U method, Phys. Rev. B 71, 035105 (2005).

[24] Phrasing more properly, this is the band representation of a spin-polarized but zero net spin state. DFT does not deal with several electron wave functions, thus it has no representation of a quantum singlet.

[25] M. D. Johannes and W. E. Pickett, Magnetic coupling between nonmagnetic ions: $\mathrm{Eu}^{3+}$ in EuN and EuP, Phys. Rev. B 72, 195116 (2005).

[26] B. J. Ruck, H. J. Trodahl, J. H. Richter, J. C. Cezar, F. Wilhelm, A. Rogalev, V. N. Antonov, B. D. Le, and C. Meyer, Magnetic state of EuN: X-ray magnetic circular dichroism at the Eu $M_{4,5}$ and $L_{2,3}$ absorption edges, Phys. Rev. B 83, 174404 (2011).

[27] D. L. Binh, B. J. Ruck, F. Natali, H. Warring, H. J. Trodahl, E.-M. Anton, C. Meyer, L. Ranno, F. Wilhelm, and A. Rogalev, Europium Nitride: A Novel Diluted Magnetic Semiconductor, Phys. Rev. Lett. 111, 167206 (2013).

[28] D. Kasinathan, K. Koepernik, and W. E. Pickett, Pressuredriven magnetic moment collapse in the ground state of $\mathrm{MnO}$, New J. Phys. 9, 235 (2007).

[29] D. Kaneko, K. Yamagishi, A. Tsukada, T. Manabe, and M. Naito, Synthesis of infinite-layer $\mathrm{LaNiO}_{2}$ films by metal organic decomposition, Physica C 469, 936 (2009). 
[30] S. Doniach, The Kondo lattice and weak antiferromagnetism, Physica B+C 91, 231 (1977).

[31] A. Luther and I. Peschel, Calculation of critical exponents in two dimensions from quantum field theory in one dimension, Phys. Rev. B 12, 3908 (1975).

[32] R. T. Scalettar, D. J. Scalapino, and R. L. Sugar, Monte Carlo simulation of the "Kondo necklace", Phys. Rev. B 31, 7316 (1985).

[33] W. Brenig, Finite-temperature properties of the two-dimensional SU(2) Kondo necklace, Phys. Rev. B 73, 104450 (2006).

[34] W. Brenig, Magnetism in the disordered two-dimensional Kondo-necklace, Int. J. Mod. Phys. 21, 2245 (2007).

[35] Y. Matsumoto, Y. Nambu, T. Honda, K. Ikeda, T. Otomo, and $\mathrm{H}$. Tageyama, High-pressure synthesis of $\mathrm{Ba}_{2} \mathrm{CoO}_{2} \mathrm{Ag}_{2} \mathrm{Te}_{2}$ with extended $\mathrm{CoO}_{2}$ planes, Inorg. Chem. 59, 8121 (2020).
[36] G.-M. Zhang, Y.-F. Yang, and F.-C. Zhang, Self-doped Mott insulator for parent compounds of nickelate superconductors, Phys. Rev. B 101, 020501 (2020).

[37] C. S. Yoo, B. Maddox, J.-H. P. Klepeis, V. Iota, W. Evans, A. McMahan, M. Y. Hu, P. Chow, M. Somayazulu, D. Häusermann, R. T. Scalettar, and W. E. Pickett, First-Order Isostructural Mott Transition in Highly Compressed MnO, Phys. Rev. Lett. 94, 115502 (2005).

[38] W. M. Li, J. F. Zhao, L. P. Cao, Z. Hu, Q. Z. Huang, X. C. Wang, Y. Liu, G. Q. Zhao, J. Zhang, Q. Q. Liu, R. Z. Yu, Y. W. Long, H. Wu, H. J. Lin, C. T. Chen, Z. Li, Z. Z. Gong, Z. Guguchia, J. S. Kim, G. R. Stewart, Y. J. Uemura, S. Uchida, and C. Q. Jin, Superconductivity in a unique type of copper oxide, Proc. Natl. Acad. Sci. USA 116, 12156 (2019). 